\title{
Un test biologique simple pour apprécier la toxicité de l'eau et des sédiments d'un puits. Toxicité comparée, in vitro, de quelques métaux lourds et de l'ammonium, vis-à-vis de trois genres de crustacés de la zoocénose des puits
}

\begin{abstract}
A simple biological test for the lethal toxicity of water and sediments of wells. Compared in vitro toxicity of some heavy metals and ammonium to three genera of aquatic crustacea living in the wells
\end{abstract}
C. Boutin(1), M. Boulanouar (2) et M. Yacoubi-Khebiza(2)
(1) Laboratoire de Zoologie et UMR CNRS 5552, Université Paul Sabatier, Bât. 4R3, 118 route de Narbonne, F-31062 Toulouse Cedex (France).
(2) Laboratoire d'Hydrobiologie, Département de Biologie, Faculté des Sciences Semlalia, BP S- 15. Marrakech (Maroc).

Résumé. - Des études antérieures ont montré que la richesse spécifique de la faune aquatique des puits de la région de Marrakech diffère de façon importante d'un puits à l'autre. Ces variations de la biodiversité des zoocénoses sont apparues corrélées avec celles de la qualité de l'eau et l'absence de stygobiontes dans certaines stations a été attribuée à la toxicité de l'eau. Afin de démontrer expérimentalement cette toxicité à l'égard de la stygofaune, la DL100 de l'eau puis celle des sédiments de 5 puits a été déterminée chez 2 genres de Crustacés stygobies fréquents dans la région, l'Amphipode Metacrangonyx spinicaudatus et l'Isopode Typhlocirolana haouzensis. Par ailleurs la $\mathrm{CL} 100 / 48 \mathrm{~h}$ de 4 ions métalliques lourds $\left(\mathrm{Cu}^{++}, \mathrm{Zn}^{++}, \mathrm{Cd}^{++}\right.$et $\mathrm{Pb}^{++}$) ainsi que des ions $\mathrm{NH}_{4}{ }^{+}$signalés dans certains des puits a été mesurée chez les 2 Crustacés stygobies, ainsi que chez un autre Isopode non stygobie, Proasellus coxalis africanus qui abonde dans certains puits de la région. La CL100 des ions testés est 40 a 60 fois plus élevée chez Typhlocirolana que chez Metacrangonyx. La CL100 de l'ammonium est nettement plus élevée chez Proasellus que chez les deux autres Crustacés; en revanche les Aselles sont plus sensibles que les Typhlocirolanes aux métaux lourds, notamment au cadmium, 
mais souvent moins sensibles que l'Amphipode. La méthodologie et ces résultats sont discutés. Ce biotest présente l'avantage de n'exiger qu'un très petit nombre d'individus (Crustacés stygobies) d'être relativement simple à mettre en œuvre, et de permettre assez rapidement - en quelques jours généralement - de détecter la toxicité globale des sédiments ou de l'eau d'une source ou d'un puits.

Mots-clés. - Stygobiologie, Biodiversité, Écotoxicologie, Métaux lourds, Crustacés.

Abstract. - The species richness of the stygofauna living in a number of wells near Marrakech is very different and former works suggest that this biodiversity is correlated with water toxicity. The LD100 of water and that of sediments from 5 wells were determined to 2 stygobiontic peracarid crustaceans common in the area: Metacrangonyx spinicaudatus (Amphipoda) and Typhlocirolana haouzensis (Isopoda). Then the $\mathrm{LC} 100 / 48 \mathrm{~h}$ of some ions which may occur in the groundwater $\mathrm{Cu}^{++}, \mathrm{Zn}^{++}, \mathrm{Cd}^{++}, \mathrm{Pb}^{++}$ and $\mathrm{NH}_{4}{ }^{*}$ was also determined to the same 2 stygobiontic crustaceans and also to an epigean aquatic isopod. Proasellus coxalis africanus which is common in some wells of the area. The CL100/48h of heavy metals to Typhlocirolana is 40 to 60 times as high than that to Metacrangonyx. The CL100/48h of ammonium is significantly higher in Proasellus than in stygobiontic crustaceans. Proasellus is more sensitive to heavy metals, especially to cadmium, than Typhlocirolana, but generally less sensitive than the Amphipoda. The experimental methodology and the different results are discussed. This bioassay offers the advantage of being relatively simple to perform with a very small number - 4 - of stygobiontic crustaceans and to display - usually in a few days the global toxicity of the sediments or of the water from a spring or a well.

Key-words. - Stygobiology, Biodiversity, Ecotoxicology, Heavy metals, Crustaceans.

\section{INTRODUCTION}

\section{Les premières études consacrées} à la faune aquatique des puits menées dans la région de Marrakech (Boutin et Boulanouar, 1983 et 1984) ont montré que la richesse spécifique et la structure des zoocénoses étaient très variables d'un puits à l'autre, et cela même en considérant des puits séparés par des distances de l'ordre du kilomètre ou même de quelques centaines de mètres seulement. Assez vite il est apparu que la richesse spécifique de la faune aquatique qui vit dans un puits, et plus particulièrement celle de la fraction stygobie de la zoocénose, c'est-à-dire de l'ensemble des espèces souterraines qui peuplent la nappe phréatique, dépend pour une grande part de la distance entre la station étudiée et les sources superficielles de pollution pouvant affecter à l'amont la nappe phréatique qui alimente la station, car cette nappe est souvent peu profonde et très vulnérable dans la région (Boutin, 1984 et 1987). Pour cette raison, une étude suivie d'un certain nombre de paramètres physico-chimiques de l'eau et des caractéristiques des phréatocénoses (abondance, richesse et diversité) a donc été conduite par l'un de nous pendant deux années, sur un ensemble représentatif de 11 
puits (Boulanouar, 1986). Cet auteur a montré que la typologie des puits décrits par des facteurs abiotiques, hydrologiques essentiellement, était la même que celle des puits caractérisés par leur faune aquatique. Dans un ensemble de stations, la dégradation croissante de la qualité de l'eau phréatique, qualité estimée à partir des paramètres physico-chimiques mesurés, semble entraîner une diminution croissante de la richesse spécifique de la zoocénose, en commençant par la réduction de la stygofaune qui disparaît totalement lorsque la station est polluée de façon importante. Des résultats très comparables ont été obtenus dans d'autres régions du Maroc où des études semblables ont pu être menées, même si les conditions climatiques, géologiques et hydrologiques ainsi que la nature des espèces stygobies présentes dans la région étaient très différentes (Boulal, 1988; Boutin et Idbennacer, 1989; Idbennacer, 1990; YacoubiKhebiza, 1990). Les espèces stygobies qui vivent habituellement dans les nappes phréatiques alluviales ou dans les aquifères karstiques apparaissent donc comme de bons marqueurs de la qualité globale de l'eau d'un puits, d'une source ou plus généralement des eaux souterraines.

Ce sont donc des observations de terrain qui ont conduit à l'idée que l'absence de certains taxons dans de nombreuses stations, pourtant situées à l'intérieur de leur aire de répartition, devait être une conséquence de la toxicité de l'eau ou des sédiments qui constituent localement l'aquifère, vis- à-vis de ces espèces. Ces observations ont également suggéré que la sensibilité des organismes stygobies, en particulier des Malacostracés, était différente d'un groupe à l'autre (Isopodes, Amphipodes, Thermosbaenacés, Syncarides), et même parfois d'une espèce à l'autre d'un même genre, comme c'est le cas chez l'Amphipode Metacrangonyx dont deux espèces, $M$. spinicaudatus et $M$. paurosexualis, sont présentes à Marrakech et dans la région, où elles cohabitent fréquemment (Boutin, 1984). Aussi a-t-il semblé nécessaire de vérifier expérimentalement au laboratoire que l'eau des puits, de même que les sédiments, prélevés dans les stations les plus pauvres faunistiquement, présentaient réellement, vis-àvis des Malacostracés stygobies de la région, une toxicité mesurable et importante, responsable de l'absence de ces espèces dans les stations les plus polluées.

Par ailleurs des études écotoxicologiques conduites par d'autres équipes travaillant dans le même secteur ont mis en évidence la présence de métaux lourds dans les sols, le sous-sol et par voie de conséquence dans la nappe (Rada, 1985; Mazlani, 1988; Sedki, 1990; Anonyme, 1991; Fars et al., 1992 et 1994; El Gharmali, 1993; Sedki et Pihan, 1994). II était donc utile d'appréhender, toujours de façon expérimentale, la toxicité relative de ces différents métaux, ainsi que celle des ions ammonium que nous avons décelés dans l'eau d'un puits particulièrement pollué, visà-vis de plusieurs genres de Malacos- 
tracés stygobies (qui vivent dans la nappe) ou non, et ainsi de comparer la sensibilité de plusieurs représentants de la faune des puits à ces diverses substances toxiques.

\section{MATÉRIEL ET MÉTHODES}

\section{La toxicité de l'eau, ainsi que} celle des sédiments prélevés dans 5 puits, ont été mesurées en déterminant la durée du séjour létale pour la totalité d'un échantillon standard d'un Crustacé stygobie (DL100) placé soit dans l'eau du puits soit dans une suspension de sédiments provenant du puits. Les 5 puits ( $P$, $, P B, P H, P 11$ et $P A)$ sont tous situés dans la zone d'épandage des eaux usés de la ville de Marrakech, à 1 ou $2 \mathrm{~km}$ au N-NW de l'agglomération (fig. 1); ils ont été choisis parce que les études antérieures (notamment celles de Boulanouar, 1986) ont montré que l'eau de ces 5 stations présente divers signes de pollution dont certains peuvent apparaître à l'occasion des analyses physico-chimiques de l'eau; la diminution de la richesse spécifique de la zoocénose de ces puits a été interprétée comme une conséquence de cette pollution. Les DL100 ont été déterminées chez deux genres de Crustacés Péracarides stygobies fréquents dans la région de Marrakech: l'Am- phipode Metacrangonyctidae Metacrangonyx spinicaudatus dont les adultes mesurent de 3 à $5 \mathrm{~mm}$ de long, et l'Isopode Cirolanidae Typhlocironana haouzensis dont les adultes atteignent et parfois dépassent $10 \mathrm{~mm}$ de long.

Les Amphipodes stygobies servant aux expériences proviennent tous d'un puits JB1 situé à peu de distance de la route nationale Marrakech Sidi Bou Othmane, à mi-chemin entre ces deux localités, sur le versant sud du massif des Jbilet, au sud-ouest du Douar Boukricha (fig. 2). Ce puits est profond et relativement protégé; il est situé loin de toute habitation et il contient une eau de bonne qualité (Boulanouar et Boutin, 1994) qui a été utilisée pour réaliser les élevages témoins. Les Isopodes stygobies proviennent du puits $P 1$ de la Palmeraie de Marrakech qui a déjà été décrit (Boutin et Boulanouar, 1983 et 1984; Boutin, 1984; Boulanouar, 1986). À environ $4 \mathrm{~km}$ au NE de la ville, ce puits se trouve à proximité de la route secondaire dite "circuit de la Palmeraie" (fig. 2); très bien protégé, il fournit une eau de très bonne qualité dans laquelle vit une faune stygobie abondante et variée.

La toxicité des ions métalliques ( $\mathrm{Cu}, \mathrm{Zn}, \mathrm{Cd}$ et $\mathrm{Pb}$ ) et de l'ammonium a été mise en évidence chez les mêmes espèces stygobies, ainsi que chez Proasellus coxalis africanus, un

Fig. 1. - Localisation des 5 puits de la Zone d'épandage des eaux usées de la ville de Marrakech, dont l'eau et les sédiments ont fait l'objet des tests de toxicité (P9, P11, PA, PB et PH). E1, E2 et E3 sont les 3 principaux émissaires amenant les eaux usées qui circulent dans des Séguias (SEU). E3 s'ouvre au milieu de la Zone d'épandange des ordures ménagères (ZEOM), qui s'étend autour de l'Usine de traitement des ordures (UTO). SEN indique le sens d'écoulement de la nappe phréatique. UNIV est l'emplacement de la Faculté des Sciences.

Fig. 1. - Location of the 5 wells in the sewage area, North of Marrakech, the water and sediments of which have been biotested for their toxicity: P9, P11, PA, PB and PH. E1, E2 and E3 are the opening of main sewers of the city; the sewage runs in small canals (seguias: SEU). E3 opens in the center of the refuse spreading area (ZEOM) where is the refuse processing factory (UTO). SEN is the direction of groundwater flowing. UNIV is the place of the Faculty of Sciences. 


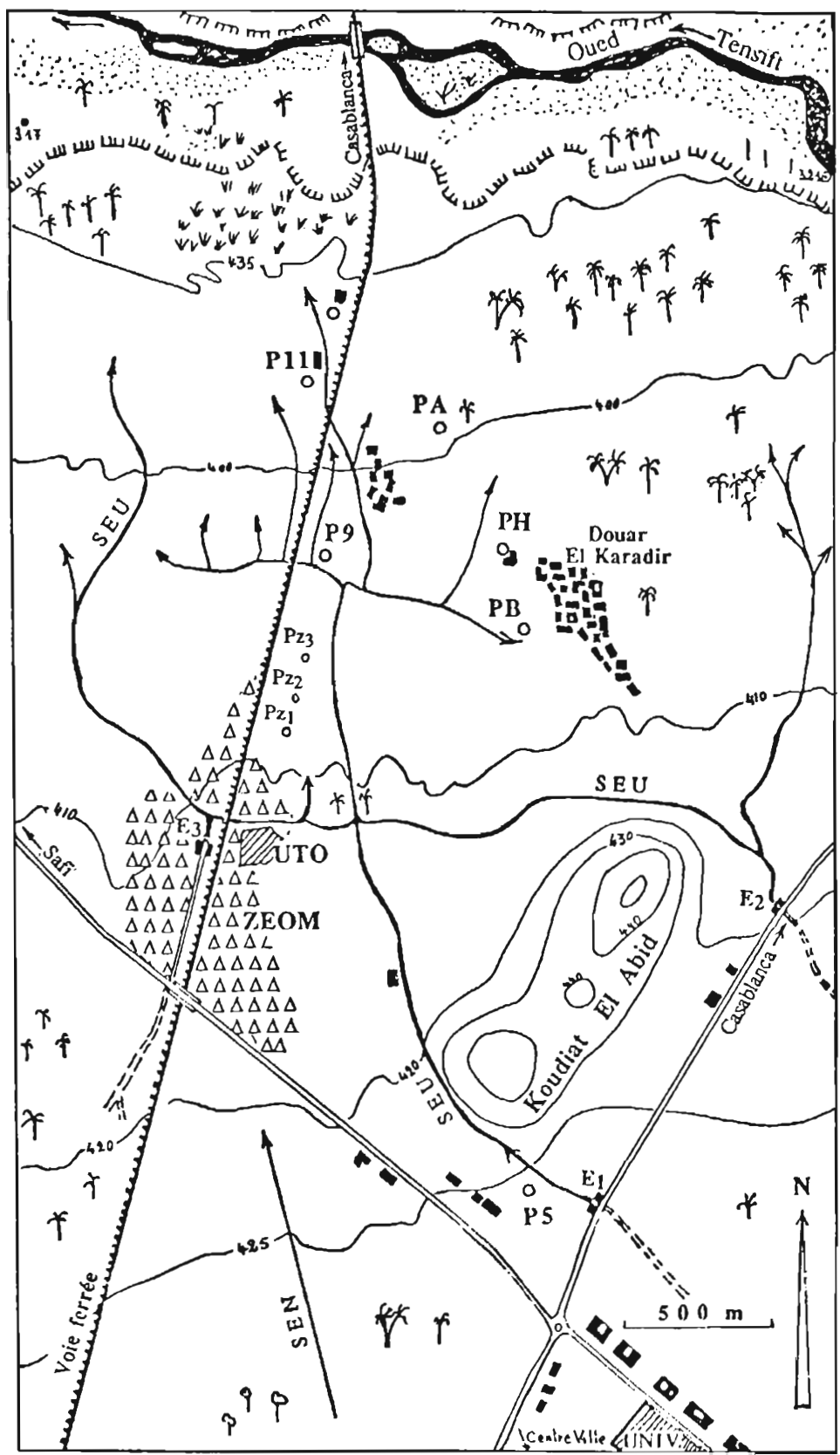




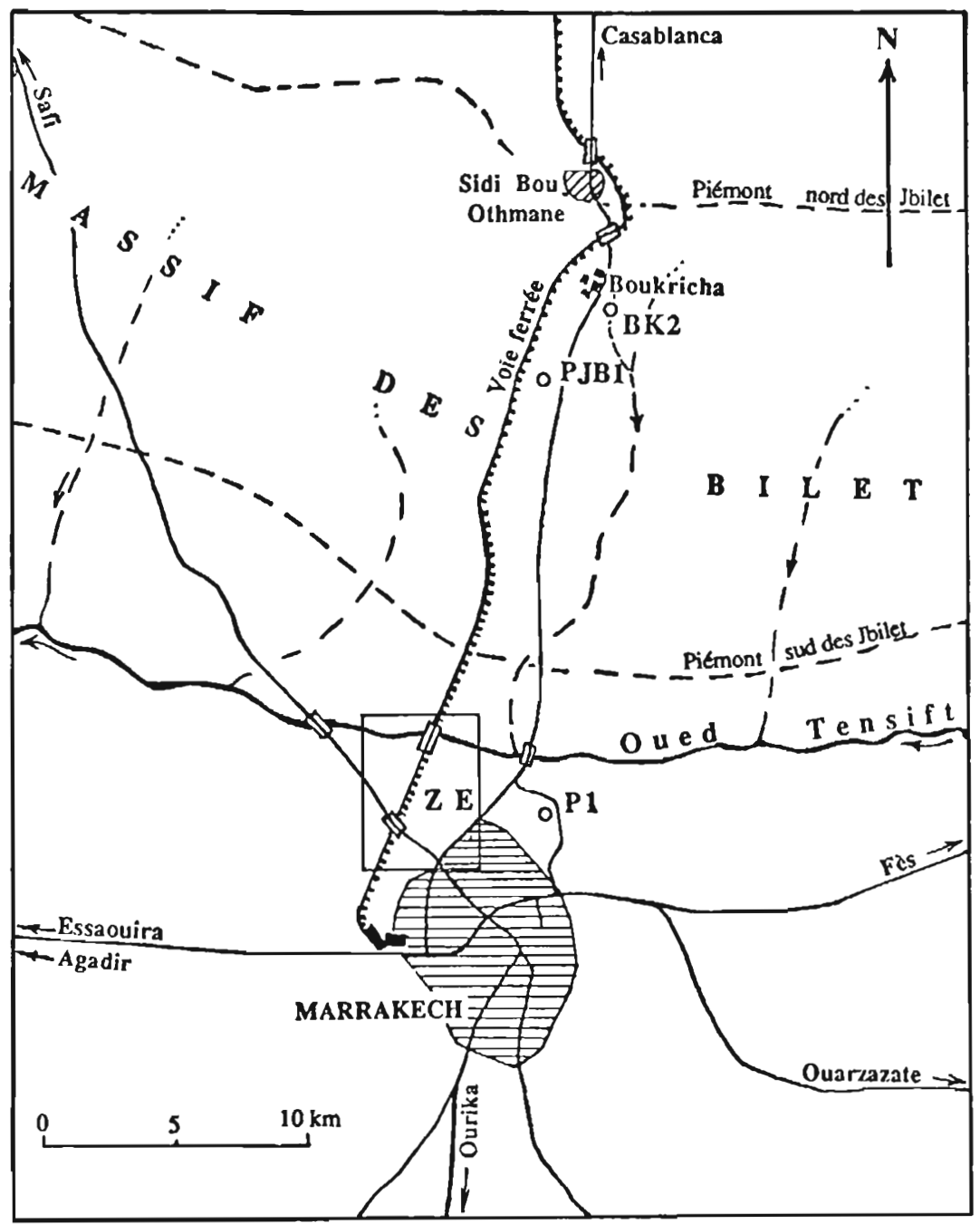

Fig. 2. - Localisation de la zone d'épandage des égouts de la Ville de Marrakech représentée Fig. 1 (ZE), et des puits BK2, PJB1 et P1 qui ont fourni respectivement les Proasellus, Metacrangonyx et Typhlocirolana utilisés au cours des expériences.

Fig. 2. - Location of the sewage area of the city of Marrakech represented in Fig. 1 (ZE) and of wells BK2, PJB1 and P1 where Proasellus, Metacrangonyx and Typhlocirolana have been captured for the experiments.

Crustacé Isopode épigé à tendances stygophiles, encore pigmenté et oculé, et qui, au nord de la chaine des Jbilet, abonde dans les puits peu profonds, non protégés et bien éclairés, où une végétation algale de Chlorophycées lui fournit substrat, abri et nourriture. Cet Asellide a été récolté 
dans le puits BK2 situé sur la rive droite d'un thalweg qui se trouve au pied du village de Boukricha déjà mentionné, entre Marrakech et Sidi Bou Othmane (fig. 2).

Toutes les expériences ont été réalisées au laboratoire sur une paillasse protégée du soleil, à une température variant entre 20 à $22^{\circ} \mathrm{C}$, proche de celle des puits de la région dont l'eau est à une température comprise entre 20 et $21^{\circ} \mathrm{C}$.

Pour déterminer la DL/100 de l'eau d'un puits, 2 flacons identiques de $100 \mathrm{ml}$, propres et rincés à l'eau distillée, sont remplis, le premier avec de l'eau filtrée provenant du puits testé, et le second qui servira de témoin, avec de l'eau filtrée prélevée dans le puits JB1. Dans chacun des 2 flacons sont placés 4 Amphipodes adultes et actifs récemment capturés à l'aide d'un filet phréatobiologique dans le puits JB1 ou, selon le cas, 4 Isopodes capturés à l'aide de nasses appâtées (Boutin et Boulanouar, 1983) au fond du puits P1. Afin d'éviter l'apparition du cannibalisme qui perturberait le bon déroulement des expériences, chaque lot de Crustacés reçoit tous les 3 jours un minuscule fragment de viande rouge (une dizaine de $\mathrm{mm}^{3}$ pour les 4 Amphipodes et une cinquantaine de $\mathrm{mm}^{3}$ pour les 4 Isopodes). L'ensemble des élevages est contrôlé quotidiennement le plus souvent (ou chaque heure dans un cas particulier où les Amphipodes ne peuvent survivre plus de 5 heures); la date des morts constatées est notée, ainsi que la durée de survie depuis le début de l'expérience, et les cada- vres sont retirés. Chaque expérience est poursuivie pendant 30 jours et au cours d'une même expérience les 5 puits sont testés simultanément afin que les résultats observés soient comparables (tab. I). Les expériences ont été répétées 5 fois avec les Amphipodes et 4 fois avec les Isopodes, à des dates différentes réparties sur l'ensemble des mois de février et mars, ceci en raison des possibilités limitées de capture des Crustacés stygobies utilisés.

\section{Pour estimer la toxicité relative} des sédiments aquifères au voisinage des 5 puits, une DL/100 a été mesurée de façon standardisée comme suit : Une série de 6 flacons identiques reçoivent chacun $100 \mathrm{ml}$ d'eau provenant du puits JB1 et 4 Crustacés, Metacrangonyx ou Typhlocirolana. Dans chacun des 5 premiers flacons on ajoute $1 \mathrm{~g}$ de sédiment fin et $\mathrm{sec}$ provenant de l'un des 5 puits étudiés, et préalablement séché pendant $24 \mathrm{~h}$ à l'étuve à $105^{\circ} \mathrm{C}$. Le sixième flacon sert de témoin. L'ensemble des élevages est nourri et surveillé comme précédemment et les durées de survie sont notées jusqu'à la mort du quatrième et dernier Crustacé de chaque flacon, si cette mort survient dans un délai inférieur ou égal à 30 ou 35 jours selon les cas, durée maximum des expériences (tab. II). Chaque série d'expériences est répétée comme précédemment et la valeur moyenne de la durée de survie dans chacun des 5 flacons est calculée.

La toxicité des métaux lourds et de l'ammonium a été déterminée en 
Tableau I. - Valeur de la DL100 de l'eau de 5 puits proches de Marrakech, pour l'Amphipode Metacrangonyx spinicaudatus et pour l'Isopode Typhlocirolana haouzensis (durée de séjour dans l'eau qui suffit, dans les conditions d'une expérience limitée à 30 jours, pour entraîner la mort de la totalité des Crustacés).

Table I. - DL100 of the water coming from 5 wells near Marrakech, to Metacrangonyx spinicaudatus (Amphipoda Metacrangonyctidae) and Typhlocirolana haouzensis (Isopoda Cirolanidae): duration of contact with water sufficient for killing all the crustaceans during a 30 days experiment.

\begin{tabular}{|c|c|c|c|c|c|c|}
\hline PUITS & P9 & PB & PH & P11 & $\mathbf{P A}$ & $\begin{array}{l}\text { témoin } \\
\text { JB1 }\end{array}$ \\
\hline \multicolumn{7}{|l|}{$\begin{array}{l}\text { DI.1100 de l'eau } \\
\text { pour Metacrangonyx }\end{array}$} \\
\hline - Nombre d'essais & 6 & 6 & 6 & 6 & 6 & 6 \\
\hline - Valeur moyenne & $4,8 h$ & $13 \mathrm{j}$ & $16 j$ & $7 \mathbf{j}$ & $10.7 j$ & $>\grave{a} 30 . j$ \\
\hline Fcart-type & $0 .+\mathrm{h}$ & $1.6 \mathrm{j}$ & $1.2 \mathrm{j}$ & $1.0 \mathrm{j}$ & $1 .+j$ & $?$ \\
\hline - Valeurs extrêmes & $+.5 \mathrm{~h}$ & $11-16 j$ & $14-18 j$ & $5-8 j$ & $9.1 .3 \mathrm{j}$ & $>\dot{\mathrm{i}} 30 \mathrm{j}$ \\
\hline \multicolumn{7}{|l|}{$\begin{array}{l}\text { DL100 de l'cau } \\
\text { pour Typhlocirolana }\end{array}$} \\
\hline - Nombre d'essais & 4 & 4 & 4 & + & $t$ & 4 \\
\hline - Valeur moyenne & $16 \mathrm{j}$ & $>\grave{a} \mathbf{3}) \mathbf{j}$ & $>\dot{a} 30 . j$ & $>$ à $30 \mathrm{j}$ & $\mathrm{j}>$ à $\mathbf{3 0}$. & $\because>$ à $30 \mathrm{j}$ \\
\hline - Ecart-type & $1.1 \mathrm{j}$ & ? & $\because$ & $?$ & $?$ & $?$ \\
\hline \multirow[t]{2}{*}{ - Valeurs extrêmes } & $15-17 \mathrm{j}$ & $>\mathrm{a} .30 \mathrm{j}$ & $>\mathrm{a} 3() \mathrm{j}$ & $>\mathrm{a} 30 \mathrm{j}$ & $>a+30 i$ & $>\dot{\mathrm{a}} 30 \mathrm{~J}$ \\
\hline & \multicolumn{6}{|c|}{ * ^ .31) jours un ('rustacés partois est mort. mais jamais plusieurs } \\
\hline
\end{tabular}

réalisant pour chaque ion envisagé des séries d'expériences successives comportant chacune de 3 à 6 flacons, 4 le plus souvent, contenant toujours au départ 4 Crustacés placés dans $100 \mathrm{ml}$ d'eau de JB1. Les essais préliminaires ont montré en effet que dans cette eau. les Crustacés stygobies restent plus actifs et survivent plus longtemps que dans de l'eau distillée ou encore de l'eau du robinet. Dans chaque flacon on ajoute un volume déterminé d'une solution mere d'un sel contenant le cation testé à une concentration connue, de façon que la concentration finale du cation testé atteigne dans le flacon une valeur allant de $0,1 \mathrm{mg} . \mathrm{I}^{-1}$ à quelques 
$\mathrm{mg} . \mathrm{I}^{-1}$ ou, à quelques dizaines ou centaines de $\mathrm{mg} \cdot \mathrm{I}^{-1}$ selon la toxicité du cation et la résistance du Crustacé utilisé pour le test. Les sels utilisés sont des sulfates de cuivre et de zinc $\left(\mathrm{CuSO}_{4}, 5 \mathrm{H}_{2} \mathrm{O}\right.$ et $\mathrm{ZnSO}_{4}$ ), des nitrates de cadmium et de plomb $\left(\mathrm{Cd}\left(\mathrm{NO}_{3}\right)_{2}\right.$ et $\left.\mathrm{Pb}\left(\mathrm{NO}_{3}\right)_{2}\right)$ enfin le chlorure d'ammonium $\mathrm{NH}_{4} \mathrm{Cl}$; des observations de terrain ont montré que les anions de ces sels semblent sans effet négatif sur la faune puisqu'ils sont parfois présents à des concentrations élevées dans certains puits dont la faune reste riche et diversifiée. Les résultats bruts d'une expérience (à savoir la mort de $0,1,3$ ou 4 crustacés au bout d'une période de 48 heures) dans chacun des 4 flacons d'une série contenant le toxique à des concentrations différentes, sont pris en compte pour choisir les concentrations qui seront utilisées dans les expériences suivantes. Ces expériences sont répétées avec des concentrations nouvelles, de façon à déterminer empiriquement la valeur minimum de la concentration du toxique qui entraine la mort des 4 Crustacés (CL100) au bout de 48 heures dans les conditions de l'expérience. Dans chaque série d'expériences, le test est répété plusieurs fois avec des concentrations du cation qui sont apparues létales en 48 heures, et avec des concentrations légèrement inférieures de façon à préciser et vérifier les premiers résultats.

\section{RÉSULTATS}

La DL100 du séjour de l'Amphipode Metacrangonyx spinicaudatus dans l'eau des 5 puits étudiés est donnée au tableau I. On voit que d'une station à l'autre elle varie de façon assez considérable puisque dans l'eau du puits PH des Amphipodes survivent généralement jusqu'à 16 jours alors que dans l'eau du puits P9 ils sont tous tués après 5 heures de séjour forcé. On retiendra que, visà-vis de $M$. spinicaudatus, P9 étant le puits dont l'eau est la plus toxique et $\mathrm{PH}$ celui dont l'eau l'est le moins, la toxicité de l'eau permet de classer les puits dans l'ordre suivant :

\section{$\mathrm{P9}>\mathrm{P} 11>\mathrm{PA}>\mathrm{PB}>\mathrm{PH}$}

Des tests identiques réalisés avec I'Isopode Typhlocirolana haouzensis confirment que l'eau du puits P9 est plus toxique que celle des autres puits, puisqu'elle est la seule à entraîner la mortalité de l'ensemble des Crustacés placés à son contact pendant la durée de l'expérimentation, limitée à 30 jours.

Les sédiments prélevés au fond et sur les parois des 5 puits étudiés contiennent également des substances toxiques, probablement solubles, qui agissent sur les Crustacés d'une façon très semblable à celle de l'eau du puits. Un gramme de sédiments secs, mis en suspension dans $100 \mathrm{ml}$ d'eau du puits témoin JB1, qui est de bonne qualité, confère à cette eau une toxicité dont la valeur apparaît au tableau II. Comme précédemment P9 est visiblement le puits le plus pollué et $\mathrm{PH}$ est celui qui l'est le moins. II est assez remarquable que le classement des 5 puits en fonction de la DL100 de leurs sédiments (ta- 
Tableau II. - Valeur de la DL100 d'une suspension de $\mathbf{1} \mathrm{g}$ de sédiments secs provenant de chacun des 5 puits étudiés, dans $100 \mathrm{ml}$ d'eau du puits témoin JB1, pour 2 Crustacés stygobies, l'Amphipode Metacrangonyx spinicaudatus et l'Isopode Typhlocirolana haouzensis, calculée à partir d'expériences ayant duré respectivement 30 et 35 jours.

Table II. - DL100 of $\mathbf{1} \mathbf{g}$ id dry well sediments dilued with $100 \mathrm{ml}$ of water from the reference well JB1, to 2 stygobiontic crustaceans, Metacrangonyx spinicaudatus (Amphipoda Metacrangonyctidae) and Typhlocirolana haouzensis (Isopoda Cirolanidae), from 30 days long and 35 days long experiments.

\begin{tabular}{|c|c|c|c|c|c|c|}
\hline PUITS & P9 & PB & PH & P11 & $\mathbf{P A}$ & témoin \\
\hline \multicolumn{7}{|c|}{$\begin{array}{l}\text { 1)I.100 des sédiments } \\
\text { pourMetacrangonyx }\end{array}$} \\
\hline - Nombre d'essais & 4 & 4 & 4 & 4 & 4 & 4 \\
\hline - Valeur moyenne & $9 \mathrm{~h}$ & $20 \mathbf{j}$ & $21 \mathrm{j}$ & $3,5 \mathbf{j}$ & $9 \mathbf{j}$ & $>\grave{a} \mathbf{3 0} \mathbf{j}$ \\
\hline - Ecart-type & $1.15 \mathrm{~h}$ & $0.8 \mathrm{j}$ & $0.8 \mathrm{j}$ & $0.6 \mathrm{j}$ & $1.8 \mathrm{j}$ & $?$ \\
\hline - Valeurs extrêmes & $8-10 \mathrm{~h}$ & $19-21 \mathrm{j}$ & $20-22$ j & $3-4 j$ & $7-11 \mathrm{j}$ & $>$ à $30 \mathrm{j}$ \\
\hline \multicolumn{7}{|c|}{$\begin{array}{l}\text { DI.100 des sédiments } \\
\text { pour Typhlocirolana }\end{array}$} \\
\hline - Nombre d'essais & 4 & 4 & 4 & 4 & 4 & 4 \\
\hline - Valeur moyenne & $3.5 \mathrm{j}$ & $>\grave{\mathbf{a}} \mathbf{3 5} \mathbf{j}$ & $>\grave{a} 35 . j$ & à $35 \mathrm{j}$ & $>$ à $35 \mathbf{j}$ & $>\grave{a} 35 \mathrm{j}$ \\
\hline - Eccart-type & $0.6 \mathrm{j}$ & $?$ & $?$ & $?$ & $?$ & $?$ \\
\hline - Valeurs extrêmes & $3-+j$ & $>\dot{a} 35 j$ & $>\grave{a} 35 \mathrm{j}$ & $>\grave{a} 35 \mathrm{j}$ & $>\grave{a} 35 \mathrm{j}$ & $>\mathrm{a} 35 \mathrm{j}$ \\
\hline
\end{tabular}

bleau II) reproduit très exactement l'ordre correspondant à celui qui a été établi précédemment à partir de la DL100 de l'eau :

$$
\text { P9 > P11 > PA > PB >PH }
$$

De ces deux séries d'expériences, il ressort clairement que la sensibilité des deux types de Crustacés stygobies utilisés pour ces tests compara- tifs est très différente, les Isopodes Typhlocirolana étant nettement plus résistants que les Amphipodes Metacrangonyx.

La toxicité globale de l'eau des 5 puits étudiés est relativement importante par comparaison avec celle de l'eau d'un puits du référence JB1 dont les caractéristiques et surtout la ri- 
Tableau III. - Valeurs de la CL/100/48h de 4 métaux lourds et de l'ammonium en mg. $\Gamma^{-1}$ pour 2 Crustacés stygobies, l'Amphipode Metacrangonyx spinicaudatus et I'Isopode Typhlocirolana haouzensis, et pour le Crustacé isopode épigé Proasellus coxalis africanus. Une valeur approchée de la CL/50/48h est également donnée à titre indicatif.

Table III. - CL/100/48h, with rough estimation of the CL/50/48h, of 4 heavy metal cations and ammonium to 3 crustaceans, the stygobiontic amphipod Metacrangonyx spinicaudatus and isopod Typhlocirolana haouzensis, and to Proasellus coxalis africanus, an epigeous Crustacea Isopoda, also living in some wells.

\begin{tabular}{|c|c|c|c|c|c|c|}
\hline $\begin{array}{l}\text { CATION } \\
\text { toxique testé }\end{array}$ & & $\mathrm{Cu}^{++}$ & $\mathrm{Zn}^{++}$ & $C \mathrm{~d}^{++}$ & $\mathrm{Pb}^{++}$ & $\mathrm{NH}_{4}^{+}$ \\
\hline \multicolumn{7}{|l|}{ CL/100 / 48 heures } \\
\hline \multirow[t]{2}{*}{ Metacrangonyx } & & $\mathbf{0 , 2 0}$ & $\mathbf{0 , 5 0}$ & 4,0 & 1,5 & 1,5 \\
\hline & $\mathrm{CL} / 50$ & $(0,15)$ & $(0,45)$ & (3 à 3,5$)$ & $(0.8)$ & $(1,4)$ \\
\hline \multirow[t]{2}{*}{ Typhlocirolana } & & 18,0 & $\mathbf{2 0 , 0}$ & 180 & 110 & 100 \\
\hline & CL 50 & $(17.0)$ & (19.5) & $(150)$ & (90) & (96) \\
\hline \multirow[t]{2}{*}{ Proasellus coxalis } & & 4,1 & 10,0 & 1,0 & 15,0 & 190 \\
\hline & $\mathrm{CL} / 50$ & $(3,5)$ & $(9,5)$ & $(0.80)$ & (1.3) & (186) \\
\hline
\end{tabular}

chesse stygobiologique suggéraient que l'eau était de bonne qualité. II apparaît également que la toxicité de l'eau est assez différente d'un puits à l'autre, ce résultat obtenu au Laboratoire ne faisant que confirmer les conclusions des études écologiques antérieures menées sur le terrain, notamment celles de Boulanouar (1986).

II apparaît aussi que les substances responsables de cette toxicité sont présentes dans les sédiments même après séchage à $105^{\circ} \mathrm{C}$, ce qui exclut les substances volatiles comme les hydrocarbures. Les substances non volatiles susceptibles d'exercer une action toxique, voire mortelle en fonction de leur concentration, sur les organismes aquatiques vivant normalement dans la nappe phréatique et dans l'eau des puits, pourraient être des micropolluants inorganiques comme des métaux lourds dont la présence a déjà été signalée, ou encore des sels d'ammonium dont nous avions décelé la présence dans plusieurs puits (Boulanouar, 1986; Boutin et Dias, 1987).

Les CL100/48 $\mathrm{h}$ des 4 métaux lourds et de l'ammonium ajoutés dans l'eau de référence provenant du 
puits JB1 figurent au tableau 3 où toutes les valeurs sont indiquées en $\mathrm{mg}$ par litre du cation testé. Chacune de ces valeurs a été vérifiée à l'issue de plusieurs séries d'expériences répétitives et peut être considérée comme exacte avec une précision d'une unité sur le dernier chiffre significatif. Au cours d'expériences préliminaires utilisant des concentrations inférieures à la $\mathrm{CL} / 100$, il a été possible d'observer une ou plusieurs fois la mort de 2 des 4 Crustacés utilisés au cours de chaque test. Ceci a permis de présenter une estimation de la CL/50 qui est donnée entre parenthèses et à titre indicatif car l'incertitude sur des valeurs obtenues 1 ou 2 fois bien souvent, et à partir de 2 individus seulement, est importante et surtout difficile à estimer.

Les valeurs des CL/100 des 5 cations testés apparaissent très différentes chez une même espèce, ce qui n'a rien d'étonnant : chez Metacrangonyx par exemple, elles vont de $0,2 \mathrm{mg} \cdot \mathrm{I}^{-1}$ pour le cuivre à $4,0 \mathrm{mg} \cdot \mathrm{I}^{-1}$ pour le cadmium; elles vont chez Proasellus de $4,1 \mathrm{mg} . \mathrm{I}^{-1}$ pour le cuivre à $190 \mathrm{mg} . \mathrm{f}^{-1}$ pour l'ammonium.

On notera également que chez les deux Crustacés stygobies la sensibilité au cuivre est peu différente mais que pour les 4 autres cations, la résistance de l'Isopode Typhlocirolana est considérablement plus grande que celle de l'Isopode Metacrangonyx. Cependant la toxicité relative des cations est sensiblement identique pour les deux Crustacés, ces cations pouvant être classés, en commençant par le plus toxique dans l'ordre suivant:

\section{$\mathrm{Cu}^{++}>\mathrm{Zn}^{++}>\mathrm{Pb}^{++}$et $\mathrm{NH}^{+}>\mathrm{Cd}^{++}$}

En revanche I'Isopode épigé Proasellus est beaucoup plus sensible au Cadmium que les Crustacés stygobies ; il présente d'une façon générale une sensibilité aux métaux lourds nettement plus grande que l'Isopode stygobie (mais plus faible que celle de l'Amphipode pour le zinc et le plomb ; il présente enfin une résistance exceptionnelle aux ions ammonium (2 fois plus grande que celle de l'Isopode stygobie et une centaine de fois plus élevée que celle de l'Amphipode). Finalement pour Proasellus, l'ordre de toxicité des ions est assez différent de celui qui s'observe chez les Crustacés stygobies bien que l'on retrouve à nouveau la succession cuivre, zinc, plomb :

$$
\mathrm{Cd}^{++}>\mathrm{Cu}^{++}>\mathrm{Zn}^{++}>\mathrm{Pb}^{++}>\mathrm{NH}^{+}
$$

\section{DISCUSSION ET CONCLUSIONS}

L'usage en écotoxicologie est d'exprimer la toxicité de l'eau ou d'un milieu en mesurant une DL/50 c'est à dire la durée de séjour qui entraine la mort de la moitié d'un échantillon animal (ou végétal) choisi pour réaliser le biotest. De la même façon la toxicité d'une substance micropolluante, organique ou inorganique, en particulier celle des métaux lourds, est habituellement déterminée en mesurant une $\mathrm{CL} / 50 / 24 \mathrm{~h}$, une $\mathrm{CL} / 50 / 48 \mathrm{~h}$ ou une CL/50/96h, c'est à dire la concentration nécessaire pour entraîner la mort - ou l'immobilisation - de la moitié de la population des organismes utilisés pour le test, au bout 
d'un temps déterminé, 24,48 ou 96 heures bien souvent. Cette pratique s'est imposée dans le protocole de nombreux tests normalisés qui utilisent généralement des organismes aquatiques de petite taille, relativement faciles à élever ou à cultiver, dont l'écotoxicologiste peut disposer en grand nombre. Chaque échantillon peut comporter un effectif de 20,50 ou 100 individus par exemple, plus encore parfois (Daphnies, microalgue etc.) quelquefois moins (Gammares), et la survie de la moitié de l'échantillon est alors significative; on l'observera généralement de façon reproductible et on appliquera un traitement statistique aux données brutes.

La présente étude n'avait pas pour objet de mettre au point un nouveau test de mesure précis et utilisable dans la pratique courante. Elle visait seulement à démontrer que l'eau de certains puits est fortement toxique, mortelle même, à l'égard de certains éléments de la faune habituellement présente dans les puits de la région en absence de pollution, et de montrer que la sensibilité des stygobiontes à divers polluants peut être très différente d'un groupe à l'autre. D'autre part il est nécessaire de préciser également qu'il est impossible de disposer simultanément d'un grand nombre de Crustacés péracarides stygobiontes en raison de la relative rareté de ces organismes et surtout de la difficulté particulière de leur capture. Quant aux possibilités d'élevage elles ne sont pas envisageables pratiquement car elles nécessiteraient une infrastructure particulière et le rendement resterait très limité en raison de la fragilité de la plupart des stygobiontes et surtout de la longueur de leur cycle vital. Bien que certains auteurs aient parfois déterminé une CL50 en utilisant des échantillons de 5 Amphipodes (Maarouf et al., 1994) nous considérons que les effectifs dont nous pouvons disposer pour chaque essai sont trop réduits pour autoriser le calcul d'une DL50 ou d'une CL50 qui soit significative. Ces raisons nous ont conduit à concevoir un plan d'expériences impliquant l'utilisation d'un petit nombre d'animaux dans chaque échantillon, mais avec répétition de chaque série d'expériences (4 ou 6 fois selon les cas) de façon que les DL100 et les CL100 observées successivement permettent le calcul d'une valeur moyenne significative.

Un des résultats du présent travail a été de montrer que la toxicité de l'eau des 5 puits considérés, de même que celle des sédiments prélevés sur ces mêmes stations, est telle, à l'égard des Crustacés stygobiontes et notamment de Metacrangonyx, qu'elle suffit à interdire la présence de ces Crustacés dans le secteur de la "zone d'épandage" où se trouvent les puits. Ainsi se trouvent confirmées les conclusions et les hypothèses proposées à l'issue des études écologiques antérieures (Boutin, 1984; Boulanouar, 1986; Boutin et Dias, 1987). L'un de nous écrivait en effet (C.B., 1984) "on est conduit à admettre que dans la région l'eau souterraine contient, au Nord de P5 (là où se trouvent les 5 puits testés aujourd'hui), une ou plusieurs substances polluantes qui rendent le milieu toxique et 
interdisent l'existence de Metacrangonyx spinicaudatus". On peut considérer que notre modeste approche écotoxicologique vient conforter les déductions de l'étude écologique de la stygofaune et de sa répartition.

Un autre résultat a été de faire apparaître que Typhlocirolana est beaucoup plus résistant que Metacrangonyx (tabs. I, II et II), ce qui constituent également une confirmation des hypothèses présentées antérieurement à partir des seules données de terrain (Boutin, 1987). Cette différence de sensibilité importante à la pollution, qui distingue les diverses espèces animales aquatiques utilisées pour réaliser des biotests, a déjà été observée très souvent, à chaque fois en fait que plusieurs modèles biologiques sont utilisés comparativement pour déterminer une CL50 ou une CL100, aussi bien chez des organismes marins (Thomas et al., 1994; Wirth et al., 1994) que chez des Crustacés d'eau douce comme les Gammares ou les Daphnies (Bluzat et Seugé, 1978; Maarouf et al., 1994).

Le fait que l'expérimentation in vitro n'ait pas fait apparaître de toxicité importante de l'eau de P11 à l'égard de Typhlocirolana, de même que les différences (très faibles comme le montre la valeur des écarts-types) entre les valeurs des DL mesurées chez Metacrangonyx à l'occasion d'essais différents renouvelés, à des dates différentes, (tab. 1 et 2) s'expliquent certainement par les variations temporelles, saisonnières en ce qui concerne Typhlocirolana, mensuelles ou quotidiennes en ce qui concerne Metacrangonyx, des teneurs en substances polluantes (voir Zauke, 1982), qui dans le cas étudié sont apportées sur la zone d'épandage par les eaux usées de la ville de Marrakech.

Les tests concernant les sédiments prélevés dans les puits montrent clairement, lorsqu'ils sont réalisés en utilisant un organisme suffisamment sensible comme Metacragonyx, que la toxicité liée aux sédiments varie dans des proportions importantes d'un puits à l'autre (tab. 2). Toutefois, bien que ces sédiments contiennent des métaux toxiques à des teneurs relativement élevées, comme l'ont montré les études antérieures citées en introduction, il est très difficile d'évaluer le rôle respectif des divers polluants présents simultanément dans un sédiment, en raison de leurs interactions synergiques complexes qui peuvent varier avec la nature du sédiment et avec l'ensemble des caractéristiques physico-chimiques de l'eau; ces difficultés apparaissent aussi bien lors de l'étude des sédiments marins que des sédiments d'eau douce (Lalande et Pinet-Alloul, 1986; Pavillon, 1994).

Le fait que les expériences réalisées avec les sédiments, n'aient pas permis, en mettant à part le cas du puits P9 plus fortement polluée, de montrer les différences entre les 4 autres puits quant à leur toxicité vis-à-vis de Typhlocirolana, est dû sans nul doute au choix, arbitraire au départ, de la quantité de sédiment choisie (1 $\mathrm{g}$ de sédiment sec) qui s'est avérée trop faible. II semble évident qu'avec plusieurs grammes de sédiment pro- 
venant de P9 dans $100 \mathrm{ml}$ d'eau, on aurait obtenu avec Typhlocirolana une DL/100 inférieure à 3,5 jours et que la toxicité des sédiments des 4 autres puits aurait pu être mesurée pendant une expérience de 30 jours. II devra être tenu compte de ces résultats lors d'expérimentations ultérieures.

Les résultats du tableau 3 confirment de façon plus précise que la résistance de Typhlocirolana est 40 à 90 fois plus grande que celle de $\mathrm{Me}$ tacrangonyx, à l'égard des 5 substances toxiques envisagées. Mais ils montrent aussi que la résistance d'un Crustacé épigé, Proasellus coxalis, peut être tantôt beaucoup plus élevée encore dans le cas des ions ammonium, tantôt au contraire intermédiaire dans le cas des métaux lourds (nettement plus faible que celle de $T y$ phlocirolana, mais bien plus grande que celle de Metacrangonyx). La taille de Metacrangonyx est nettement plus petite que celle des deux autres Crustacés, mais comme il appartient à l'Ordre des Amphipodes alors que Typhlocirolana et Proasellus sont des Isopodes la taille n'est peut-être pas le seul facteur en cause.

On pourrait être tenté de comparer la sensibilité de Metacrangonyx, évaluée ici pour la première fois, avec celle d'un autre Amphipode dulçaquicole. Maarouf (1993) a mesuré les CL50 $24 \mathrm{~h}$ du Cuivre et du Zinc pour Gammarus gauthieri et obtenu respectivement 0,028 et $1,5 \mathrm{mg} . \mathrm{l}^{-1}$ alors que les CL50 $48 \mathrm{~h}$ que nous avons estimées $\left(0,15\right.$ et $\left.0,45 \mathrm{mg} . \mathrm{I}^{-1}\right)$ avec ces mêmes métaux semblent indiquer que Metacrangonyx résisterait mieux que le Gammare à la présence du Cuivre mais serait en revanche nettement plus sensible au Zinc. Toutefois ces valeurs n'ayant pas été obtenues dans les mêmes conditions expérimentales la comparaison n'a guère de signification et les différences observées ne fournissent que des indications ne permettant aucune conclusion définitive.

On notera que Proasellus appartient à une famille d'Isopodes dulçaquicoles épigés et que de nombreuses espèces de la famille, vivent encore dans des eaux de surface, courantes ou stagnantes, et prolifèrent là où la matière organique d'origine végétale abonde; la décomposition de cette matière végétale peut conduire à des teneurs non négligeables en sels azotés dont ceux d'ammonium. II convient en outre de savoir que dans un même environnement des Crustacés Malacostracés appartenant à divers groupes taxonomiques fixent différemment les métaux lourds (Zauke et Petri, 1993) et montrent des teneurs très différentes avec une "considérable interspecific heterogeneity without consistent dependence" (Petri et Zauke, 1993).

On peut également noter une sensibilité au Cadmium relativement très forte chez Proasellus, dont la CL/100 est 4 fois plus faible que celle de $\mathrm{Me}$ tacrangonyx. II semble que les Isopodes dulçaquicoles Asellidés, soient plus sensibles au Cadmium que les Isopodes marins (Jones, 1975; Braginskiy et Shcherban 1978; Williams et al., 1985; Martin et Holdich, 1986) sans que l'on sache pourquoi. 
Et l'on peut aussi considérer que les Metacrangonyctidae, comme les Cirolanidae, sont des Crustacés "limnostygobiontes thalassoïdes " c'est-à-dire des formes actuellement inféodées aux eaux douces souterraines et continentales, mais sans proches parents dans les eaux douces de surface, et directement dérivées d'ancêtres marins qui ont d'abord colonisé activement les milieux interstitiels ou karstiques littoraux avant de devenir continentaux de façon passive, à l'occasion des régressions marines passées, pendant la seconde phase d'un "modèle biphase d'évolution biologique et biogéographique" (Boutin et Coineau, 1990; Coineau et Boutin, 1992; Boutin, 1993 et 1994). Metacrangonyx spinicaudatus est une des espèces les plus "primitives" du genre, une de celles qui a conservé le plus de ressemblances morphologiques avec les lointains ancêtres marins de la famille (Messouli, 1988 et 1994; Boutin, 1993 et 1994) et il se trouve que nous avons pu observer (C.B., inédit), que cette espèce a aussi conservé une grande tolérance aux très fortes salinités, qui pourrait être en rapport avec les mécanismes d'accumulation et d'excrétion des ions, y compris les métaux toxiques.

Si les résultats obtenus posent au moins autant de questions qu'ils n'en résolvent concernant la diversité de la sensibilité des Crustacés, stygobies ou non, aux quelques substances chimiques testées, ils fournissent cependant, comme nous l'avons souligné précédemment, la vérification de plusieurs des hypothèses formulées à l'issue des études écologiques intéressant la stygofaune de la région.

Enfin la méthode utilisée au cours de cette étude présente l'avantage d'être relativement simple à mettre en œuvre à partir d'un échantillon biologique de petite taille ( 4 individus pour l'essai et autant pour le témoin suffisent) ; elle fournit un résultat assez rapide, en quelques jours le plus souvent, et on peut l'appliquer aussi bien à l'eau, ou aux sédiments d'une source comme à ceux d'un puits.

Cependant, compte tenu de la diversité de la sensibilité des espèces aux divers polluants et en particulier aux métaux lourds, le "diagnostic sommaire de la qualité de l'eau " que nous avons déjà proposé (Boutin, 1984 et 1987, Boulanouar, 1986), prenant en compte non pas les réactions d'une espèce particulière mais la richesse spécifique de l'ensemble de la zoocénose du puits, ainsi que sa richesse "stygobiologique", nous apparaît plus apte à déceler la détérioration de la qualité globale de l'eau d'une source ou d'un puits, surtout lorsque la nature de la pollution susceptible d'affecter le point d'eau est inconnue.

\section{REMERCIEMENTS}

Les auteurs tiennent à remercier les deux lecteurs anonymes qui ont contribué par leurs observations et suggestions, à améliorer la qualité du manuscrit, ainsi que la Rédaction de Hydroécologie Appliquée qui a accepté une mise à jour de la bibliographie prenant en compte des publications postérieures au dépôt du texte. 


\section{BIBLIOGRAPHIE}

Anonyme 1991. Rapport de la SAFEGE : Étude de l'assainissement de la Ville de Marrakech; étude des usages de l'eau et des rejets, pollution et qualité des eaux. $130 \mathrm{pp}$.

Bluzat R. \& Seugé J., 1978. Toxicité aiguë comparée de 5 produits détersifs sur 4 espèces d'invertébrés habitant les eaux douces. C.R. Acad. Sc. Paris, 286 série D : 1391-1394.

Boulal M., 1988. Recherches écologiques sur la faune aquatique des puits de la région de Tiznit (Anti-Atlas occidental, Maroc). Thèse $3^{\text {e }}$ Cycle, Fac. Sc. Univ. Marrakech, $228 \mathrm{p}$.

Boulanouar M., 1986. Étude écologique comparée de quelques puits de la région de Marrakech. Impact des pollutions sur la zoocénose des puits. Thèse $3^{\theta}$ Cycle, Univ. Marrakech, $159 \mathrm{pp}$.

Boutin C., 1984. Sensibilité à la pollution et répartition de quelques espèces de Crustacés phréatobies à Marrakech (Maroc occidental). Mém. Biospéol., $11(38)$ : 55-64.

Boutin C., 1987. L'eau des nappes phréatiques superficielles, une richesse naturelle vitale mais vulnérable. L'exemple des zones rurales du Maroc. Sciences de l'Eau, 6(3) : 357-365.

Boutin C., 1993. Biogéographie historique des Crustacés Malacostracés stygobies du Maroc. Thèse Doct. Univ. Claude-Bernard, Lyon I, $263 \mathrm{p}$.

Boutin C., 1994. Phylogeny and biogeography of Metacrangonyctid Amphipods in North Africa. Hydrobiologia, 887: 49-64.

Boutin C. \& Boulanouar M., 1983. Méthodes de capture de la faune stygobie: Expérimentation de differents types de pièges appâtés dans les puits de Marrakech. Bull. Fac. Sc. Marrakech (Sect. Sc. Vie), 2 : 5-21.
Boutin C. \& Boulanouar M., 1984. Premières données sur la faune des puits des environs de Marrakech (Maroc occidental). Verh. Intern. Verein. Limnol., 22 : 1762-1765.

Boutin C. \& Coineau N., 1990. "Regression model ", "Modèle biphase d'évolution" et origine des Micro-organismes stygobies interstitiels continentaux. Rev. Micropal. 33(3-4) : 303-322.

Boutin C. \& Dias N., 1987. Impact de l'épandage des eaux usées de la ville de Marrakech sur la nappe phréatique. Bull. Fac. Sc. Marrakech (Sect. Sc. Vie), $3: 5-25$.

Boutin C. \& Idbennacer B., 1989. Faune stygobie du sud de l'Anti-Atlas marocain: premiers résultats. Rev. Sc. de l'eau, 2 : 891-904.

Braginskiy L.P. \& Shcherban E.P., 1978. Acute toxicity of heavy metals to aquatic invertebrates at different temperatures. Gidrobiol. Zh., 14 : 86-92.

Chandler T., Coull B. \& Davis J., 1994. Sediment and Aqueous Phase Fenvalerate Effects on Meiobenthos: Implications for Sediment Quality Criteria Development. Marine Environ. Res., 37 : 313-327.

Coineau N. \& Boutin C., 1992. Biological processes in space and time. II, Colonization, Evolution and Speciation in Interstitial Stygobionts. In: The Natural History of Biospeleology, Camacho A.I. ed., Monografias VII. Mus. Nac. Cien. Nat. and CSIC, Madrid: 425-451.

El Gharmali A., 1993. Étude de l'action des vers de terre sur la biodisponibilité des métaux lourds, ( $\mathrm{Cu}, \mathrm{Zn}, \mathrm{Cd}, \mathrm{Pb}$ ) dans les sols de la zone d'épandage des eaux usées de la Ville de Marrakech. Thèse $3^{\mathrm{e}}$ Cycle, Fac. Sc. SemIalia, Univ. Marrakech, 148 pp.

Fars S., Sedki A. \& Nejmeddine A., 1992. Le devenir des métaux lourds dans le sol et le sous-sol du champ d'épandage de la Ville de Marrakech. Act. Coll. Intern. Hydrogéol. milieux discontinus sous climats arides., Marrakech 22-25 avril 1992, 276-279. 
Fars S., Sedki A. \& Nejmedine A., 1994. Efficacité du lagunage anaérobie sous climat aride dans l'élimination des métaux lourds (Cu, $\mathrm{Zn}, \mathrm{Cd}$ et $\mathrm{Cr})$. Rev. Fac. Sc. Marrakech, $8: 71-82$.

Idbennacer B., 1990. - Recherches écologiques, biogéographique et démographiques sur la faune aquatique souterraine de la région de Guelmin (sud-ouest de l'Anti-Atlas marocain). Thèse de $3^{e}$ Cycle, Fac. Sc. Univ. Marrakech : $275 \mathrm{pp}$.

Jones M.B., 1975. Synergistic effects of salinity, temperature and heavy metals on mortality and osmoregulation in marine and estuarine isopods (Crustacea). Mar. Biol. $30: 13-20$.

Lalande M. \& Pinel-Alloul R., 1986. Acute toxicity of Cadmium, Copper and Zinc to Tropocyclops prasinus mexicanus (Cyclopoida, Copepoda) from three Quebec lakes. Environ. Toxicol. and Chem., 5 : 95-102.

Maarouf A., 1993. Toxicité létale et bioaccumulation du Zinc respectivement chez deux macro-invertébrés d'eau douce: Gamamrus gauthieri et Dreissena polymorpha. Optimisation de deux biotests: tests de toxicité létale chez G. gauthiery et test de bioaccumulation à moyen terme chez D. polymorpha. Thèse Doctorat d'État, Fac. Sc. Meknès (Maroc) : 1-296.

Maarouf A., Mazlani S., Pihan J.-Cl. \& Belkhadir E., 1994. Comparaison de la sensibilité de deux Crustacés: Gammarus gauthieri et Daphnia magna, à la toxicité létale des extraits de sédiments de quatre cours d'eau marocains. Annls Limnol. 30(3): 197-207.

Martin T.R. \& Holdich D.M., 1986. The acute lethal toxicity of heavy metals to peracarid crustaceans (With particular reference to fresh-water asellids and gammarids). Wat. Res., 20(9) : 1137 1147.

Mazlani S., 1988. Présence et évaluation de l'écotoxicité par bioessais des métaux lourds dans les eaux usées de la Ville de Marrakech (Maroc); suivi de l'épuration en lagunage expérimental. Thèse $3^{e}$ Cycle, Fac. Sc. Univ. Marrakech, $84 \mathrm{pp}$.

Messouli M., 1988. Les Crustacés Amphipodes souterrains du Groupe Metacrangonyx: Répartition, Systématique et Phylogénie. Mém. Thèse de $3^{\mathrm{e}} \mathrm{Cy}$ cle, Fac. Sc. Univ. Marrakech, 234 p.

Messouli M., 1994. Evolution, Phylogénie et Biogéographie historique des Metacrangonyctidae, Crustacés Amphipodes stygobies du Nord de l'Afrique et des régions voisines. Thèse Doctorat d'État, Fac. Sc. Univ. Marrakech, $309 \mathrm{p}$.

Pavillon J.-F., 1994. Les problèmes d'évaluation de la toxicité des sédiments: problèmes fondamentaux et recherches. Oceanis, 20(3) : 55-67.

Petri G. \& Zauke G.-P., 1993. Trace Metals in Crustaceans in the Antarctic Ocean. Ambio, 22(8) : 529-536.

Rada A., 1985. Écotoxicologie des métaux lourds, mise au point bibliographique et contamination métallique de l'Oued Tensift (région de Marrakech). Thèse $3^{e}$ Cycle, Fac. Sc. Univ. Marrakech, 113 pp.

Sedki A., 1990. Étude de la contamination par les métaux lourds de l'écosystème terrestre dans la zone d'épandage des eaux usées de la ville de Marrakech. Thèse $3^{\ominus}$ Cycle, Fac. Sc. Marrakech, $133 \mathrm{pp}$.

Sedki A. \& Pihan H.C., 1994. Les métaux lourds chez les vers de terre vivant dans les sols du champ d'épandage des eaux usées de la ville de Marrakech. Rev. Fac. Sc. Marrakech, 8 : 8391.

Wirth E., Chandler T., DiPinto L. \& Bidleman T., 1994. Assay of Polychlorinated Biphenyl Bioaccumulation from Sediments by Marine Benthic Copepods Using a Novel Microextraction Technique. Environ. Sci. Technol., 28(9) : 1609-1614.

Yacoubi-Khebiza M., 1990. Écologie et biogéographie des biocénoses aquatiques 
des nappes alluviales de quelques vallées du haut-Attas de Marrakech (Maroc). Paléobiogéographie des Crustacés phréatobies. Thèse $3^{\theta}$ Cycle, Fac. Sc. Univ. Marrakech: 246 pp.

Zauke G.P., 1982. Cadmium in Gammaridae (Amphipoda, Crustacea) of the rivers Werra and Weser. II, Seasonal variation and correlation to tempera- ture and other environmental variables. Water Res., 16: 785-792.

Zauke G.P. \& Petri G., 1993. Metal concentration in Antarctic Crustacea: The problems of background levels. In : Ecotoxicology of Metals in Invertebrates. Dallinger R. \& Rainbow P.S. eds. CRC Press Inc., Boca Raton, FL, USA, 73-101. 